\title{
Cooperative Enclosing and Grasping of an Object by Decentralized Mobile Robots Using Local Observation
}

\author{
Yuichi Kobayashi · Shigeyuki Hosoe
}

Accepted: 1 October 2011 / Published online: 27 October 2011

(C) The Author(s) 2011. This article is published with open access at Springerlink.com

\begin{abstract}
This paper discusses the design of a decentralized capturing behavior by multiple mobile robots. The design is based on a gradient descent method using local information. The task of capturing a target is divided into two subtasks; the enclosing subtask and the grasping subtask. An analysis of the convergence of the local control policy in the enclosing subtask is provided, while ensuring that the neighborhood relation of the robot system is preserved. In the grasping subtask, the force-closure condition in decentralized form is used to design a local objective function. A local estimation of the shape of the object is introduced so that each robot can decide how to move on the basis of only the available local information. The proposed local control policies were evaluated using simulations and the flexibility of the system was verified owing to the decentralized nature of the system. The enclosing subtask was implemented using multiple mobile robots with local observation from omnidirectional CCD cameras.
\end{abstract}

Keywords Decentralized control · Multiple robots . Cooperation $\cdot$ Force-closure

\section{Introduction}

The decentralized and self-organizing behavior of creatures (e.g., flocking behavior of birds [15]) has gained a lot of interest in a variety of research areas. From an engineering

Y. Kobayashi $(\bowtie)$

Department Electrical and Electronic Engineering, Tokyo University of Agriculture and Technology, Tokyo, Japan

e-mail: yu-koba@cc.tuat.ac.jp

S. Hosoe

RIKEN-TRI Collaboration Center, Nagoya, Japan viewpoint, the decentralized behavior of creatures inspired the design of flexible and adaptive artificial systems (e.g., the decentralized control of multi-legged robots $[8,19])$. If we can design multiple robot systems (such as multiple mobile robots for navigation [2], soccer robots [6], multi-agent decision making [18], collision avoidance [1], pushing an object [22] and foraging [20]) which emulate such behaviors, we can expect several advantages:

- The system will be less expensive in terms of observation and communication since each robot in the system is controlled by only local information.

- The system will be sufficiently robust to allow changes to the number of elements, specifically, the changes in which the elements fail.

- The system will adapt to the environment without centralized observation or computation.

Roumeliotis et al. proposed decentralized position control by integrating observed information using a Kalman filter [16]. Desai et al. proposed formation control of multiple robots using a non-holonomic constraint [5]. Lin et al. analyzed a pursuit task using distributed multiple mobile robots [9]. With respect to a so-called sensor-network, which aims to measure object information by mobile sensing robots, Cortes et al. produced a coverage control strategy that can guarantee the convergence and the correctness of the algorithm [4]. Chung et al. proposed a target tracking behavior using multiple mobile robots [3]. Task allocation among multiple robots has been also discussed by Ulam et al. [20] and Mataric et al. [11].

With regard to capturing objects, Song et al. proposed a potential-based formation control of multiple mobile robots on the object [17]. Yamaguchi proposed a distributed, smooth, time-varying feedback control law for the hunting behavior of multiple non-holonomic mobile robots [24]. 
Hsieh et al. proposed a pattern generation method using a position-based constraint between multiple robots [7]. Pereira et al. proposed the notion of "object closure", where the object cannot escape in any direction by the existence of robots, while considering the geometrical position of the robots and the object [14]. The behavior of robots that achieves object closure and then moves the object to a specific position is called "caging" behavior.

In this paper, we propose the decentralized control of multiple mobile robots in order to capture a (moving) target object based on only local observation excluding any explicit communication among the robots. In the proposed framework, no integrated sensor information is used since this generally requires some communication among the robots, as used in $[3,16]$. With caging behaviors or by placing robots along the object designed in $[7,14,17]$, the geometrical information of the object is known and used to calculate the configuration space. In the approach of grasping behavior proposed in this paper, the shape of the object is assumed to be unknown and can be measured only locally. This assumption has the advantage of reducing the cost of observation and communication of each robot. By omitting the non-holonomic constraints of mobile robots, the control law proposed in this paper is simpler than the feedback control law proposed with non-holonomic constraints [5, 24]. With these omissions, it is still possible to achieve the enclosing behavior using real robots with two wheels, as experimentally demonstrated in this paper.

First, we will discuss the enclosing behavior design with decentralized control. With respect to the grasping behavior, it is shown that the objective function for the total system can be decentralized while relating the control of the grasping behavior to the control of the enclosing behavior. The proposed control policy is applied to an object with a convex but unknown shape. We use local approximation of the object shape to address this problem.

The task of capturing an object by multiple mobile robots is described in Sect. 2, accompanied by a discussion on the design method requirements of the decentralized system. The proposed motion control methods are described in Sects. 3 and 4, corresponding to the problem definitions. Both methods are evaluated by simulations in Sect. 5. Section 6 experimentally implements and verifies the enclosing behavior using real multiple mobile robots.

\section{The Capturing Task}

The capturing task of a moving object is divided into two subtasks. The first subtask is to enclose the target object while approaching it. The second subtask is to find the optimal configuration of robots required to grasp the object by moving along the surface of the object. Some common assumptions for these two problems are described below:

- Each robot is regarded as a point that can move in any direction with a limited speed.

- Each robot senses the positions of its neighboring robots.

- Each robot acts on the basis of the local sensor information.

- The total number of robots is unknown to each individual robot.

- Each robot does not communicate with any of the other robots.

- There is no obstacle in the environment.

A one-dimensional structure is introduced for the configuration of the robot system where each robot recognizes only (a maximum of) two robots as neighbors as shown in Fig. 1. We now go on to describe the individual formulations of the enclosing and the grasping problems.

\subsection{The Enclosing Problem}

The goal of the robot system is to surround an object while approaching it.

- The shape of the target object is a circle with known radius $R$.

- The target moves randomly, but its speed is slower than that of the robots.

- Each robot senses the position (center) of the object.
Fig. 1 Enclosure problem with 1-D topology and polar coordinate system

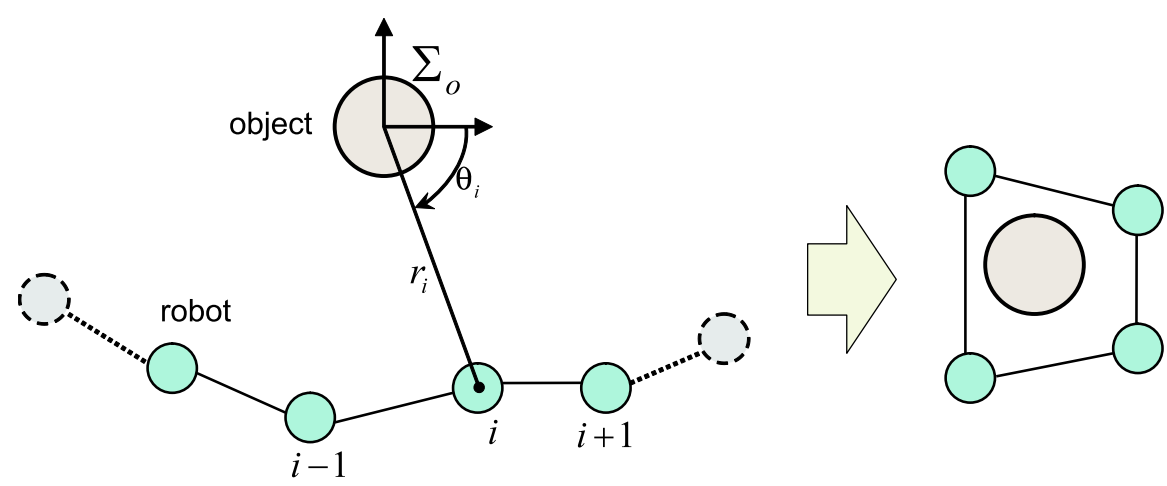


The configuration of the robot system is represented in Fig. 1 by a graph. Each robot is expressed as a node and a link between these nodes indicates that the nodes connected by the link are the neighbor nodes. The structure (neighborhood relation) of the robot system changes, in general, from an open-chain form to a circular form, as shown in Fig. 1. At the beginning of a task, the ends of the chain system are not connected because of the large distance between them, as shown on the left of Fig. 1. After sometime, however, the system forms a circle when both ends come close enough to recognize each other as neighbors, as shown on the right of Fig. 1. We assume that the shape of the object (a circle) and the radius $(R)$ are known by the robots so that in the enclosing task, priority is given to the analysis of the basic nature of the decentralized control. In addition, we assume that each robot cannot precisely identify the shape of the object and thus approximates the object to be circular when it has not enclosed the object (that is, it is not close enough to the object) because of the low accuracy of actual sensors. After sometime, it becomes easier to identify the shape of the object when each robot comes close enough to the object. In the next section, we consider the unknown factors in identifying the shape of the object while discussing the grasping task.

\subsection{The Grasping Problem}

The goal of the grasping task is to determine an optimal configuration that does not allow the object to escape from the robots. In this task, it is assumed that all of the robots already touch the surface of the object.

- The object does not move until the optimal configuration is determined.

- The shape of the object is convex.

- Each robot senses the normal directions of the object surface at its own position and also at the position of the neighboring robots. ${ }^{1}$

- After every small movement, each robot does not leave the surface of the object.

Instead of knowing the overall shape of the object, it is assumed that each robot can obtain local and partial information of the object's shape from the positions and directions of its neighboring robots as indicated in Fig. 2. For irregular object shapes, the normal direction of the robot on edges cannot be uniquely determined. Considering real robots with volume, it is assumed that the robot can change its position continuously on the edge of the object as shown in Fig. 3. In simulations, this assumption is expressed by replacing an edge with a small curve.

\footnotetext{
${ }^{1}$ So that each robot can observe its neighboring robots contacting on the surface of the object, we assume that the height of robots is higher than that of the object.
}

Fig. 2 Robot on object surface
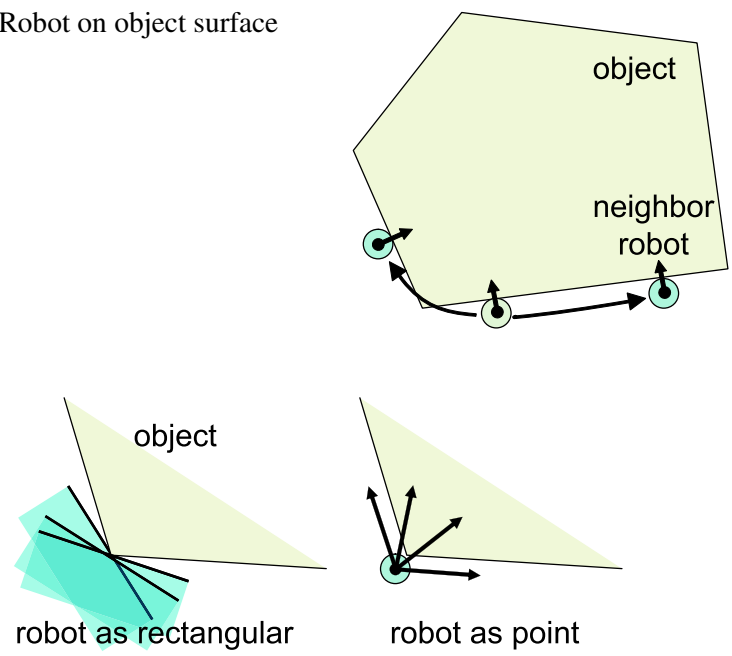

Fig. 3 Assumption on posture on edge

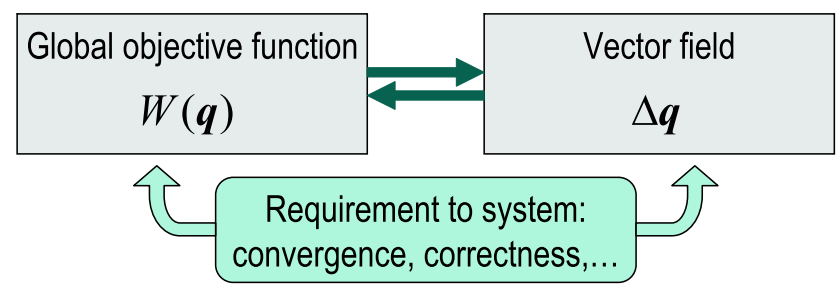

Fig. 4 Design of autonomous decentralized system

\subsection{Motion Control and Evaluation Function}

Let $q_{i} \in \mathbb{R}^{2}, i=1, \ldots, n$ be a state vector of robot $i$. One way to design decentralized behavior is to first determine a potential function $W$, that corresponds to a global evaluation function, and then derive control $\Delta q_{i}$ so that the minimum value of $W$ is eventually attained (cf. [25]). A second way is to directly design $\Delta q_{i}$ (see Fig. 4). In this research, the second way is followed, and a control law for enclosing behavior is proposed and analyzed. Then, the relation of this law with an evaluation function is discussed in Sect. 3. On the basis of the observation in this section, the design of a control law for grasping behavior is presented in Sect. 4.

\section{Decentralized Enclosing Behavior}

The polar coordinate system is used to design the enclosing behavior. As shown in Fig. 1, the position of robot $i$ is expressed as $\left(r_{i}, \theta_{i}\right)$ in the object coordinate $\Sigma_{O}$. With this polar coordinate system, the neighboring robots $i_{+}$and $i_{-}$of robot $i$ are determined according to the following equations:

$i_{+}=\arg \min _{j}\left|\theta_{j}-\theta_{i}\right|, \quad 0<\theta_{j}-\theta_{i}<\theta_{\max }$ 
Fig. 5 Transition from multiple groups with chain forms to a circular form

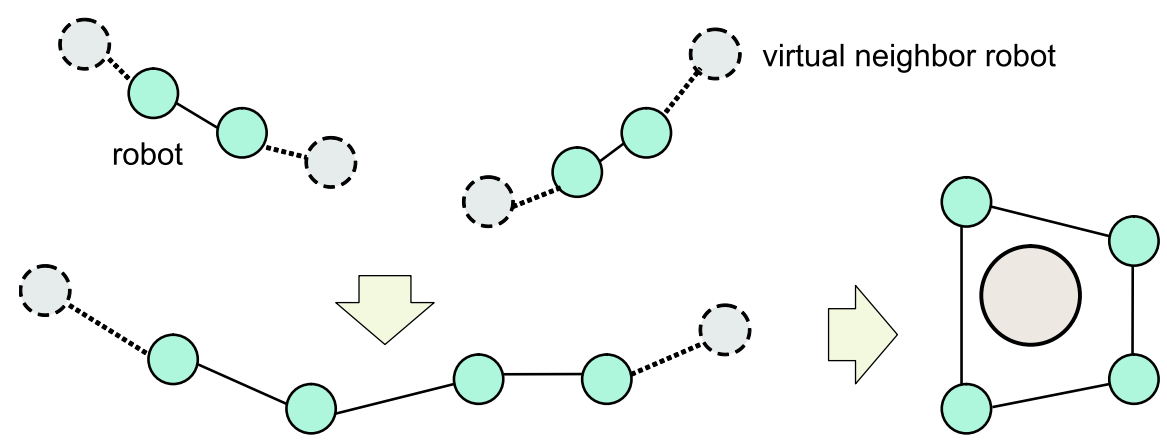

$i_{-}=\arg \min _{j}\left|\theta_{j}-\theta_{i}\right|, \quad 0>\theta_{j}-\theta_{i}>-\theta_{\max }$,

where $\theta_{\max }$ denotes the threshold value for neighbor recognition. $^{2}$ To simplify the presentation, it is assumed that $\theta_{i} \neq \theta_{j}$ for all $i \neq j$. Thus, $i_{+}$and $i_{-}$are uniquely determined if they exist. If a robot does not detect any neighboring robots, it does not move in $\theta$ direction, that is, $\dot{\theta}=0$.

Note that based on the assumption that each robot only observes the positions of its neighbors within its own coordinates, robot $i$ cannot observe $\theta_{i}, \theta_{i_{+}}, \theta_{i_{-}}$. Instead, it can obtain $\left(\theta_{i_{+}}-\theta_{i}\right)$ and $\left(\theta_{i_{-}}-\theta_{i}\right)$, which is described in detail in Sect. 6.

With the neighborhood relation defined above, the following two cases arise: (i) The robot system forms a set of several open chains (see upper left part of Fig. 5). (ii) $n$ robots make a closed chain (circular structure) surrounding the object (see right part of Fig. 5). In case (i), robots at the edge nodes of the chain will be called edge robots. In both cases, by renumbering, it is assumed that non-edge robot $i$ has robots $i+1$ and $i-1$ as its neighbors and $\theta$ 's are arranged such that $\theta_{1}<\cdots<\theta_{n}$ holds. In this paper, the number of open chains is assumed to be one for simplicity. When determining the movements of edge robots (; let them be robots 1 and $n$ ), their virtual neighbors 0 and $n+1$ need to be introduced. Their $\theta$ values are defined by:

$\theta_{0}=\theta_{1}-\theta_{\max }$,

$\theta_{n+1}=\theta_{n}+\theta_{\max }$.

The same notations $\theta_{0}$ and $\theta_{n+1}$ are also used in the circular case, but in this case they should be understood as

$\theta_{0}=\theta_{n}-2 \pi, \quad \theta_{n+1}=\theta_{1}+2 \pi$.

\footnotetext{
${ }^{2}$ Note that we can consider uncertainty of sensing through this threshold value. If the sensors of a robot provide reliable observation only when its neighboring robot is close to it, we define small value of $\theta_{\max }$.
}

The enclosing behavior aims (1) to achieve a robot configuration such that all the robots surround the object with an equal interval (2) without changing the order of the robots, guaranteeing that the structure transforms from an open chain system to a circular system. Owing to the definition of the neighborhood, and to realize the enclosing behavior, it is necessary that

$n>2 \pi / \theta_{\max }$,

i.e., the number of robots must be greater than $2 \pi / \theta_{\max }$. This will be assumed throughout the paper. The two issues mentioned above are discussed in the following sections.

\subsection{Convergence with Circular Structure}

This section describes how the robot system converges to the desired configuration in the circular structure case, partly following our earlier discussion in $[9,21] .{ }^{3}$ The behavior of the radius component is designed as follows:

$\dot{r}_{i}(t)=-a\left(r_{i}(t)-R\right), \quad a>0$.

Obviously, the radius component converges to $r_{i}(t)=R$ by the equation. The behavior of the angular component is designed as follows:

$$
\begin{gathered}
\dot{\theta}_{i}(t)=-b\left(\theta_{i-1}(t)-2 \theta_{i}(t)+\theta_{i+1}(t)\right), \\
i=1, \ldots, n, b>0,
\end{gathered}
$$

where $\theta_{0}$ and $\theta_{n+1}$ are defined by (3.5). Note that on the basis of this equation, each robot can decide $\dot{\theta}_{i}(t)$ using only the relative information of $\theta_{i+1}(t)-\theta_{i}(t)$ and $\theta_{i-1}(t)-$ $\theta_{i}(t)$. This equation can be rewritten in matrix form as

$$
\dot{\boldsymbol{\theta}}=A \boldsymbol{\theta}+\boldsymbol{d}, \quad \boldsymbol{\theta}=\left[\theta_{1}, \ldots, \theta_{n}\right]^{T}
$$

\footnotetext{
${ }^{3}$ Note that researches $[9,21]$ are relevant to the issue discussed in Sect. 3.1. They do not refer to the issues described in the following Sects. 3.2, 3.3, and 3.4.
} 
$A=-b\left[\begin{array}{ccccccc}-2 & 1 & 0 & \cdots & 0 & 0 & 1 \\ 1 & -2 & 1 & 0 & \cdots & 0 & 0 \\ & & & \ddots & & & \\ 0 & 0 & \cdots & 0 & 1 & -2 & 1 \\ 1 & 0 & 0 & \cdots & 0 & 1 & -2\end{array}\right] \in \mathbb{R}^{n \times n}$

$\boldsymbol{d}=b[-2 \pi, 0, \ldots, 0,2 \pi]^{T} \in \mathbb{R}^{n}$.

It is easy to show that the following holds true:

$\lim _{t \rightarrow \infty} \theta(t)=q v+\boldsymbol{r}$,

where $\boldsymbol{v}=[1, \ldots, 1]^{T} \in \mathbb{R}^{n}$ and

$q=\frac{1}{n} \sum_{i=1}^{n} \theta_{i}(0)-\frac{1}{n-1} n \pi, \quad \boldsymbol{r}=\frac{1}{2 \pi} n[0,1, \ldots, n-1]^{T}$.

First, define $\overline{\boldsymbol{\theta}}$ by $\overline{\boldsymbol{\theta}}=q \boldsymbol{v}+\boldsymbol{r}$. Then, it is clear that

$A \overline{\boldsymbol{\theta}}+\boldsymbol{d}=\mathbf{0}, \quad \overline{\boldsymbol{\theta}} \equiv q \boldsymbol{v}+\boldsymbol{r}$.

Thus, $\overline{\boldsymbol{\theta}}$ is an equilibrium of (3.9). In addition, note the fact that the eigenvalues of the circulant matrix

$C=\left[\begin{array}{cccc}c_{1} & c_{2} & \cdots & c_{n} \\ c_{n} & c_{1} & \cdots & c_{n-1} \\ & & \vdots & \\ c_{2} & c_{3} & \cdots & c_{1}\end{array}\right] \in \mathbb{R}^{n \times n}$

can be expressed as follows:

$\lambda_{i}=c_{1}+c_{2} \omega^{i-1}+\cdots+c_{n} \omega^{(i-1)(n-1)}, \quad i=1, \ldots, n$,

where $\omega$ denotes the $n$-root of 1 . Using this equation, the eigenvalues of $A$ are given as

$\lambda_{i}=2 b\left\{\cos \left(2 \pi \frac{1}{i-1} n\right)-1\right\}, \quad i=1, \ldots, n$.

Thus, all the eigenvalues except $\lambda_{1}$ are negative when $b>0$ and therefore $(\boldsymbol{\theta}-\overline{\boldsymbol{\theta}})$ converges to the null space of $A$ corresponding to $\lambda_{1}=0$, that is, $(\boldsymbol{\theta}-\overline{\boldsymbol{\theta}})$ converges to $\{\boldsymbol{q} \mid \boldsymbol{q}=$ $p \boldsymbol{v}, p \in \mathbb{R}\}$. In addition, $\boldsymbol{v}^{T} \boldsymbol{\theta}=\sum_{i} \theta_{i}$ must be constant because $\frac{d}{d t} \boldsymbol{v}^{T} \boldsymbol{\theta}(t)=0$. This completes the proof of (3.10).

\subsection{Preservation of Order}

This section shows that the order among robots does not change under the control of (3.8) in both the circular structure and the open chain structure cases. First, the circular case is considered. Contrary to what is to be proved, assume at instant $t$, robots $i$ and $i+1$ change their order. At this instant, the following holds true:

$\theta_{1}(t)<\cdots<\theta_{i}(t)=\theta_{i+1}(t)<\cdots<\theta_{n}(t)$.
On the other hand, the angle difference between the two robots satisfies the following:

$\dot{\theta}_{i+1}-\dot{\theta}_{i}=\left\{\begin{array}{c}b\left\{3\left(\theta_{1}-\theta_{2}\right)+2 \pi+\theta_{3}-\theta_{n}\right\}, \\ i=1 \\ b\left\{3\left(\theta_{i}-\theta_{i+1}\right)+\left(\theta_{i+2}-\theta_{i-1}\right)\right\}, \\ 2 \leq i \leq n-2 \\ b\left\{3\left(\theta_{n-1}-\theta_{n}\right)+2 \pi+\theta_{1}-\theta_{n-2}\right\}, \\ i=n-1\end{array}\right.$

In any case, the first term on the right hand of (3.16) is zero while the last terms are positive, owing to (3.15). Thus, the following holds true:

$\left.\frac{d}{d t}\left(\theta_{i+1}(t)-\theta_{i}(t)\right)\right|_{\theta_{i}(t)=\theta_{i+1}(t)}>0$.

By continuity, this equation must also be satisfied just before the instant $t$. However, this obviously contradicts (3.15). It can be similarly confirmed that the order between $\theta_{1}$ and $\theta_{n}$ is also preserved.

For an open chain form, the corresponding differential equation is

$\dot{\theta}_{i+1}-\dot{\theta}_{i}=\left\{\begin{array}{c}b\left\{3\left(\theta_{1}-\theta_{2}\right)+\theta_{\max }+\theta_{3}-\theta_{1}\right\}, \\ i=1 \\ b\left\{3\left(\theta_{i}-\theta_{i+1}\right)+\left(\theta_{i+2}-\theta_{i-1}\right)\right\}, \\ 2 \leq i \leq n-2 \\ b\left\{3\left(\theta_{n-1}-\theta_{n}\right)+\theta_{\max }+\theta_{n}-\theta_{n-2}\right\}, \\ i=n-1\end{array}\right.$

and the preservation of the order can be similarly proved.

The preservation of the order can be proved by similar procedures even if the values of $b$ are different among adjacent robots. The convergence of the system can also be proved using the property of compartment matrices. That is, the properties of convergence and order preservation are guaranteed even if the speeds of robots are different owing to hardware constraints.

\subsection{Transition from Open Chain to Circular Form}

In this section, the robot system transition from open chain form to circular form is discussed. In the case of open chain, the evolutionary equations can be expressed as

$\dot{\theta}_{1}(t)=b\left(\theta_{2}(t)-\theta_{1}(t)-\theta_{\max }\right)$,

$\dot{\theta}_{n}(t)=b\left(\theta_{n-1}(t)-\theta_{n}(t)+\theta_{\max }\right)$.

By introducing the following difference system:

$\Delta_{1}=\theta_{2}-\theta_{1}, \quad \Delta_{2}=\theta_{3}-\theta_{2}, \ldots, \Delta_{n-1}=\theta_{n}-\theta_{n-1}$, 
$(3.21)$

the evolution of the open chain system can be rewritten as

$$
\dot{\Delta}_{i}= \begin{cases}-2 \Delta_{1}+\Delta_{2}+\theta_{\max }, & i=1 \\ -2 \Delta_{i}+\Delta_{i-1}+\Delta_{i+1}, & 2 \leq i \leq n-2 \\ -2 \Delta_{n-1}+\Delta_{n-2}+\theta_{\max }, & i=n-1 .\end{cases}
$$

Let $\boldsymbol{\Delta}=\left[\Delta_{1}, \ldots, \Delta_{n-1}\right]^{T}$ and $S=\left\{\boldsymbol{\Delta} \in \mathbb{R}^{n-1} \mid 0 \leq \Delta_{i} \leq\right.$ $\left.\theta_{\max }, i=1, \ldots, n-1\right\}$. First, it will be shown that $S$ is an invariant set, i.e., any trajectory that starts from $S$ stays in $S$.

For $i=1, \ldots, n-1$, let $S_{i, 0}$ and $S_{i, \theta_{\max }}$ be defined as $S_{i, 0}=\left\{\boldsymbol{\Delta} \in \mathbb{R}^{n-1} \mid \Delta_{i}=0,0 \leq \Delta_{j} \leq \theta_{\max }, j \neq i\right\}$ and $S_{i, \theta_{\max }}=\left\{\boldsymbol{\Delta} \in \mathbb{R}^{n-1} \mid \Delta_{i}=\theta_{\max }, 0 \leq \Delta_{j} \leq \theta_{\max }, j \neq i\right\}$, respectively. Let us consider trajectories that can go out of $S$ at boundary $S_{i, 0}$. When $\boldsymbol{\Delta} \in S_{i, 0}$, it can be seen that

$$
\begin{array}{ll}
\dot{\Delta}_{1}=\Delta_{2}+\theta_{\max } \geq 0, & i=1 \\
\dot{\Delta}_{i}=\Delta_{i-1}+\Delta_{i+1} \geq 0, & 2 \leq i \leq n-2 \\
\dot{\Delta}_{n-1}=\Delta_{n-2}+\theta_{\max } \geq 0, & i=n-1,
\end{array}
$$

which means that the trajectory does not go out of $S$ in the negative direction of $\Delta_{i}$. When $\boldsymbol{\Delta} \in S_{i, \theta_{\max }}$, it can be seen that

$$
\begin{array}{ll}
\dot{\Delta}_{1}=\Delta_{2}-\theta_{\max } \leq 0, & i=1 \\
\dot{\Delta}_{i}=-2 \theta_{\max }+\Delta_{i-1}+\Delta_{i+1} \leq 0, & \\
\quad 2 \leq i \leq n-2 & \\
\dot{\Delta}_{n-1}=\Delta_{n-2}-\theta_{\max } \leq 0, & i=n-1,
\end{array}
$$

which means that the trajectory does not go out of $S$ in the positive direction of $\Delta_{i}$. This proves that $S$ is an invariant set.

Next, we will prove that the open chain form transforms to the circular form by assuming the contrary. That is, we assume that the system keeps the open chain form. (3.22) can be rewritten in matrix form as

$$
\dot{\boldsymbol{\Delta}}=-B \boldsymbol{\Delta}+\boldsymbol{h}, \quad \boldsymbol{h}=\left[\begin{array}{c}
\theta_{\max } \\
0 \\
\vdots \\
0 \\
\theta_{\max }
\end{array}\right],
$$$$
B=\left[\begin{array}{cccccc}
2 & -1 & 0 & \cdots & & \\
-1 & 2 & -1 & 0 & \cdots & \\
& & & \ddots & & \\
& \cdots & 0 & -1 & 2 & -1 \\
& & \cdots & 0 & -1 & 2
\end{array}\right] \text {. }
$$

The stability of matrix $-B$ is shown in Appendix. Using this, the difference system (3.22) converges to the solution of $-B \boldsymbol{\Delta}+\boldsymbol{h}=0$, that is, equilibrium $\boldsymbol{\Delta}=[1, \ldots, 1]^{T} \theta_{\max }$. Therefore, assuming the preservation of the open chain form, the system converges to the equilibrium $\boldsymbol{\Delta}=$ $[1, \ldots, 1]^{T} \theta_{\max }$. Let $\sigma$ denote the angle between robot $n$ and 1 on the side of the open jaw, that is,

$\sigma=2 \pi-\sum_{i=1}^{n-1} \Delta_{i}$

After convergence, it follows from the assumption $n>$ $2 \pi / \theta_{\max }$ that

$\sigma=2 \pi-(n-1) \theta_{\max }<n \theta_{\max }-(n-1) \theta_{\max }=\theta_{\max }$,

which contradicts the assumption of the preservation of the open chain form. Thus, the transition from the open chain form to the circular form is proven.

\subsection{Local Control and Evaluation Function}

It has been shown that the local control law (3.8) can achieve the convergence to the desired state without changing the one-dimensional structure. In addition, the open-chain system is guaranteed to transform to the circular system. An interpretation of this control law can be given from an objective function viewpoint. Let us define the total objective function $W$ by

$W=\sum_{i=1}^{n} f\left(r_{i}, \theta_{i}, \theta_{i+1}\right)$,

where $f$ are local objective functions given by

$f\left(r_{i}, \theta_{i}, \theta_{i+1}\right)=\frac{1}{2} \alpha\left(r_{i}-R\right)^{2}+\frac{1}{2} \beta\left(\theta_{i+1}-\theta_{i}\right)^{2}$.

By considering the gradient of $W$, the following control laws that are respectively equivalent to (3.7) and (3.8) can be derived:

$\Delta r_{i}=-\eta \frac{\partial W}{\partial r_{i}}=-\eta \alpha\left(r_{i}-R\right)$,

$\Delta \theta_{i}=-\eta \frac{\partial W}{\partial \theta_{i}}=-\eta \beta\left(\theta_{i}-\frac{\theta_{i+1}+\theta_{i-1}}{2}\right)$.

The second term in (3.33) will also be used to design the grasping behavior.

\section{Decentralized Grasping Behavior Design}

In this section, we consider the grasping task for an object with an unknown shape. A decentralized control will be derived on the basis of the localized the force-closure condition and the local approximation of the object shape. First, we derive the local evaluation function, and then propose a local control policy by introducing the local approximation of the object shape. 


\subsection{Decentralized Form of Evaluation Function}

It is extensively discussed in robotics literature how a robot can be used to firmly grasp an object [12]. The goal of the grasping behavior is to design a robot configuration that does not allow the object to escape in any direction. This goal is closely related to the notion of force-closure. In this study, it is assumed that the contact between a robot and the object is a point contact without friction.

Definition 1 (force-closure) Let $F_{e} \in \mathbb{R}^{6}$ be an external wrench given to the object. A grasp is called force-closure if and only if there exists a contact force $\boldsymbol{f}_{c} \in F C=\{\boldsymbol{f} \in$ $\left.\mathbb{R}^{k} \mid f_{i} \geq 0\right\}$ for any external force $F_{e}$ satisfying

$-F_{e}=G \boldsymbol{f}_{c}$,

where $G$ denotes the grasp matrix that transforms a contact force to the object coordinate.

In the case of contact without friction, the grasp matrix $G$ with contact points can be written as

$G=\left[\begin{array}{ccc}\boldsymbol{n}_{1} & \ldots & \boldsymbol{n}_{k} \\ \boldsymbol{p}_{1} \times \boldsymbol{n}_{1} & \ldots & \boldsymbol{p}_{k} \times \boldsymbol{n}_{k}\end{array}\right]$,

where $\boldsymbol{p}_{i}$ denotes the position of robot $i$ in a fixed object coordinate and $\boldsymbol{n}_{i}$ denotes the normal vector to the object surface at $\boldsymbol{p}_{i}$. To check whether the force-closure condition is satisfied, the following theorem can be used:

Theorem 1 (force-closure condition [12]) The following two conditions are equivalent:

- The grasp is force-closure.

- The convex hull constructed by column vectors of $G$ contains the neighborhood of the origin.

The convex hull constructed by set of vectors $S=\left\{\boldsymbol{v}_{i} \mid i=\right.$ $1, \ldots, k\}$ is defined as

ConvexHull $(S)=\left\{\boldsymbol{v}=\sum_{i=1}^{k} a_{i} \boldsymbol{v}_{i}: \sum_{i=1}^{k} a_{i}=1, a_{i} \geq 0\right\}$.

In the grasping task described in this paper, the translational motion of the object will be focused on, since any rotational motion of the object is less important for capturing. Thus, the grasp matrix becomes $G=\left[\boldsymbol{n}_{1}, \ldots, \boldsymbol{n}_{n}\right] \in \mathbb{R}^{2 \times n}$. Corresponding to $\boldsymbol{n}_{i}$, angle parameters $\phi_{i}$ are introduced by $\phi_{i}=\arctan \left(n_{i 2} / n_{i 1}\right)$, that is, angles between $\boldsymbol{n}_{i}$ and vector $[1,0]$.

Then, assuming that the robots on the object surface are numbered counter-clockwise by the convexity of the object they satisfy:

$\phi_{i}<\phi_{i+1}, i=1, \ldots, n-1, \quad \phi_{n}<\phi_{1}+2 \pi$.
Fig. 6 Decentralized

force-closure condition

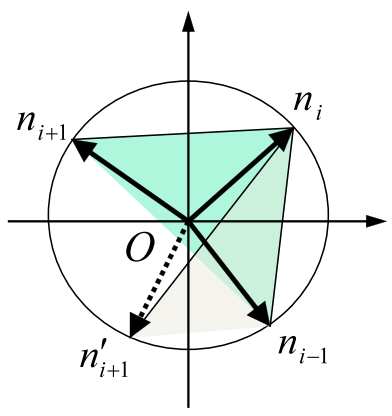

Furthermore, the second condition in the theorem can be rewritten in a decentralized form as follows:

$\phi_{i+1}-\phi_{i}<\pi, \quad i=1, \ldots, n-1$.

$\phi_{1}-\phi_{n}<-\pi$.

The implication of these conditions are much more easily understood by referring to Fig. 6 . In the figure $\left(\boldsymbol{n}_{i-1}, \boldsymbol{n}_{i}, \boldsymbol{n}_{i+1}\right)$ contains the origin as an interior point, while $\left(\boldsymbol{n}_{i-1}, \boldsymbol{n}_{i}, \boldsymbol{n}_{n+1}^{\prime}\right)$ does not.

On the basis of the above considerations, local evaluation functions are defined as:

$g\left(\phi_{i+1}, \phi_{i}\right)=\left(\phi_{i+1}-\phi_{i}\right)^{2}-\pi^{2}$.

Obviously, they take negative values if and only if (4.4) and (4.5) hold true. Thus, on them, the total objective function is defined as

$W_{G}=\sum_{i=1}^{n} g\left(\phi_{i+1}, \phi_{i}\right)$

The gradient descent for this $W_{G}$ is derived as

$\Delta \phi_{i}=-\eta \frac{\partial W_{G}}{\partial \phi_{i}}=-\eta\left(\phi_{i}-\frac{\phi_{i+1}+\phi_{i-1}}{2}\right)$.

Note that (4.8) is exactly the same as (3.35) in Sect. 3. Therefore, if $\phi_{i}$ are adjusted according to (4.8), then the controlled system benefits from the following advantages:

- The order is maintained, given that the neighborhood relation is defined exactly in the same way as in (3.1) and (3.2), i.e., just by replacing $\theta_{i}$ and $\theta_{\max }$ in (3.1) and (3.2) by $\phi_{i}$ and $\phi_{\max }$, respectively.

- Each robot can judge whether the force-closure condition is achieved by locally examining the conditions $g\left(\phi_{i+1}, \phi_{i}\right)<0$.

For the manipulation of robot hands, there are many studies on how to best determine contact points by introducing grasp evaluation functions [10, 27]. Liu et al. proposed using the radius of the minimum hyper sphere that contacts the convex hull as a grasp evaluation function [10]. This evaluation function relies on the idea that the grasp can be more 
stable when the configuration is far from the boundary of the force-closure condition, which means it is more difficult to break the force-closure condition. The minimum of $W_{G}$ corresponds to the maximum of evaluation functions discussed in $[10,27]$.

\subsection{Local Approximation of Object Shape}

In this study, note that $\Delta \phi_{i}$ cannot be controlled directly in applications. Instead, this should be achieved by allowing robots to move along the surface of the object and change their positions according to the normal direction of the surface. To achieve this, the positions of the robots have to be related to their changes in orientation. As described below, this is achieved by locally approximating the object shape using the neighboring information.

As shown in Fig. 7, the local shape of the object is approximately parameterized as

$\boldsymbol{p}_{i}(u)= \begin{cases}\boldsymbol{v}_{1 p}^{i} u+\boldsymbol{v}_{2 p}^{i} u^{2}, & 0 \leq u \leq 1, \\ \boldsymbol{v}_{1 n}^{i} u+\boldsymbol{v}_{2 n}^{i} u^{2}, & -1 \leq u \leq 0,\end{cases}$

where $\boldsymbol{v}_{1 \bullet}^{i}, \boldsymbol{v}_{2 \bullet}^{i} \in \mathbb{R}^{2}$ denote the parameters for the curve (• denotes either $p$ or $n$ ). By differentiating (4.9a) and (4.9b), we obtain

$\frac{\partial \boldsymbol{p}_{i}(u)}{\partial u}=\boldsymbol{v}_{1 \bullet}^{i}+2 \boldsymbol{v}_{2 \bullet}^{i} u$,

which gives the direction of the curve at $\boldsymbol{p}_{i}$. The curve should be perpendicular to the direction of robot $i$ at $u=0$. This condition can be expressed as

$\left[\frac{\partial \boldsymbol{p}_{i}(0)}{\partial u}\right]^{T} \boldsymbol{n}_{i}=0, \quad \boldsymbol{n}_{i}=\left[\begin{array}{ll}1 & 0\end{array}\right]^{T}$.

Let $x_{j}^{i} \in \mathbb{R}^{2}$ denote the position of robot $j$ in the coordinate system of robot $i$. The conditions that both ends of the curve coincide with the neighboring robots $i+1$ and $i-1$ can be expressed as

$$
\begin{aligned}
& p_{i}(-1)=x_{i-1}^{i} \\
& p_{i}(1)=x_{i+1}^{i} .
\end{aligned}
$$

Similarly, let $\boldsymbol{n}_{j}^{i} \in \mathbb{R}^{2}$ denote the unit vector that expresses the direction of robot $j$ in the coordinate system of robot $i$. The condition that the curve is perpendicular to the directions of neighboring robots $i+1$ and $i-1$ at both the ends can be expressed as

$$
\begin{aligned}
& {\left[\frac{\partial \boldsymbol{p}_{i}(-1)}{\partial u}\right]^{T} \boldsymbol{n}_{i-1}^{i}=0} \\
& {\left[\frac{\partial \boldsymbol{p}_{i}(1)}{\partial u}\right]^{T} \boldsymbol{n}_{i+1}^{i}=0}
\end{aligned}
$$

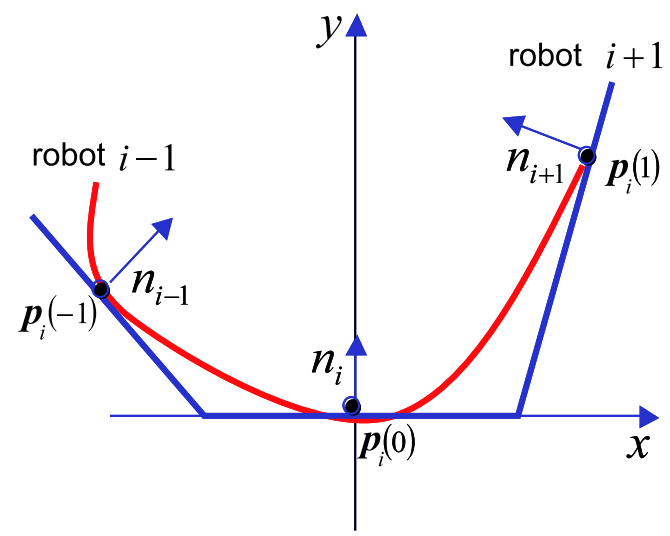

Fig. 7 Local approximation of object shape

respectively. For $u \geq 0,(4.11),(4.13)$, and (4.15) can be represented in matrices form as

$\left[\begin{array}{ll}\boldsymbol{v}_{1 p}^{i} & \boldsymbol{v}_{2 p}^{i}\end{array}\right]\left[\begin{array}{ccc}\boldsymbol{I} & \boldsymbol{n}_{i} & \boldsymbol{n}_{i+1}^{i} \\ \boldsymbol{I} & O_{2 \times 1} & 2 \boldsymbol{n}_{i+1}^{i}\end{array}\right]=\left[\begin{array}{ll}\boldsymbol{x}_{i+1}^{i} & O_{1 \times 2}\end{array}\right]$.

From this, the parameters $\boldsymbol{v}_{1 p}^{i}, \boldsymbol{v}_{2 p}^{i}$ can be determined as

$\left[\begin{array}{ll}\boldsymbol{v}_{1 p}^{i} & \boldsymbol{v}_{2 p}^{i}\end{array}\right]=\left[\begin{array}{ll}\boldsymbol{x}_{i+1}^{i} & O_{1 \times 2}\end{array}\right]\left[\begin{array}{ccc}I & \boldsymbol{n}_{i} & \boldsymbol{n}_{i+1}^{i} \\ I & O_{2 \times 1} & 2 \boldsymbol{n}_{i+1}^{i}\end{array}\right]^{-1}$

The curve parameters for $u \leq 0$ can be similarly obtained as

$\left[\begin{array}{ll}\boldsymbol{v}_{1 n}^{i} & \boldsymbol{v}_{2 n}^{i}\end{array}\right]=\left[\begin{array}{ll}\boldsymbol{x}_{i-1}^{i} & O_{1 \times 2}\end{array}\right]\left[\begin{array}{ccc}I & \boldsymbol{n}_{i} & \boldsymbol{n}_{i-1}^{i} \\ I & O_{2 \times 1} & 2 \boldsymbol{n}_{i-1}^{i}\end{array}\right]^{-1}$

using (4.12) C (4.14) and (4.11).

When the desired change in the robot direction $\Delta \phi_{i}$ is obtained using (4.8), this change of direction can be expressed using the curve parameters and $u$ as

$\tan \left(\Delta \phi_{i}\right)=\frac{v_{12}^{i}+2 v_{22}^{i} u}{v_{11}^{i}+2 v_{21}^{i} u}$

The solution to this equation for $u$ is obtained as

$\tilde{u}=-\frac{\tan \left(\Delta \phi_{i}\right) v_{11}^{i}-v_{12}^{i}}{2\left(\tan \left(\Delta \phi_{i}\right) v_{21}^{i}-v_{22}^{i}\right)}$

Thus, the displacement of robot $i$ that realizes the change of direction $\Delta \phi_{i}$ is expressed by

$\dot{\boldsymbol{x}}_{i}=\boldsymbol{p}(\tilde{u}) / \Delta t$,

where $\Delta t$ denotes a sampling time step for controlling a robot. 


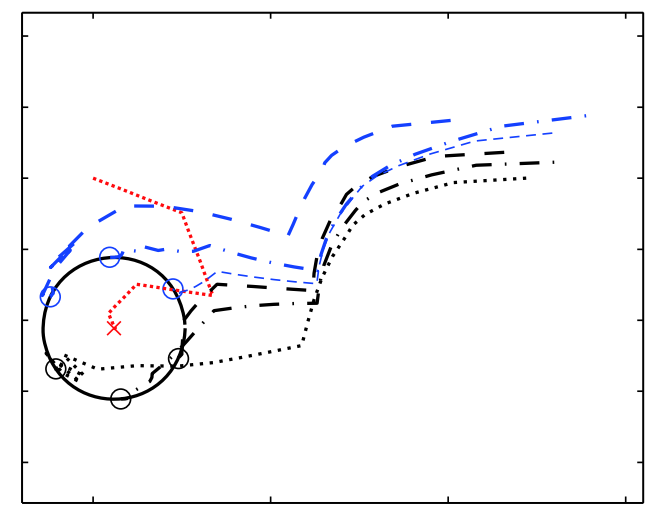

Fig. 8 Trajectories in enclosure problem

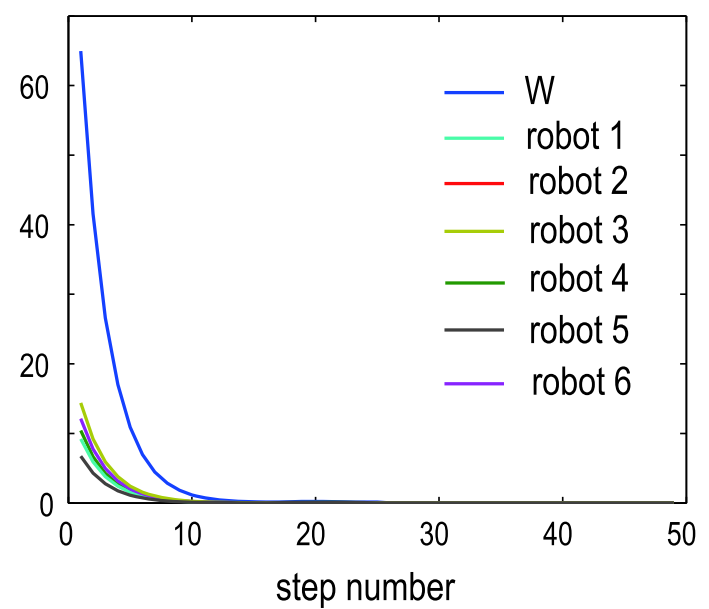

Fig. 9 Values of objective functions

\section{Simulation}

In this section, the proposed local control policy for the enclosing task and the grasping task is verified by evaluating the potential function.

\subsection{Enclosing Problem}

Figure 8 shows an example of robot trajectories where the circular target moves randomly with robot number $n=6$ and threshold value $\theta_{\max }=\pi / 2$. The values of the total objective function $W$ and local objective functions $f_{i}$ evaluated along the trajectories are shown in Fig. 9. It can be seen that both the trajectories of the robots and the total evaluation function converge. With all of different initial positions of the robots, the robots finally achieved the enclosing task as far as the speed of the object is lower than that of the robots.

\subsection{Grasping Problem}

The proposed local control policy for the grasping task is tested with $n=4$. Figure 10 shows an example of trajectories of robots with an ellipsoidal object. The robots are

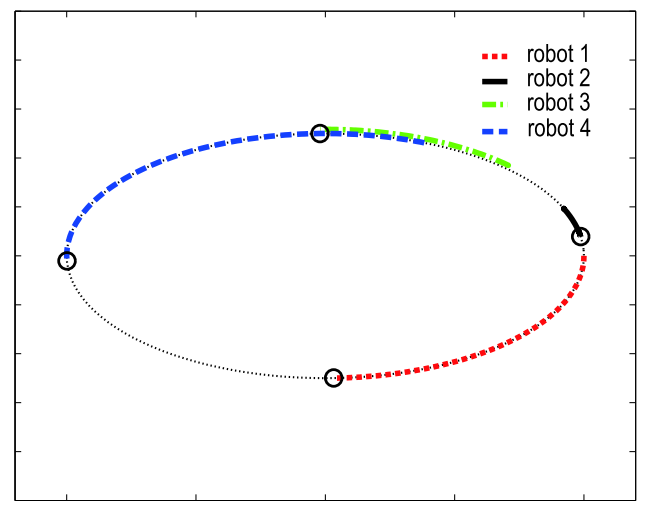

Fig. 10 Trajectories of robots for grasping problem

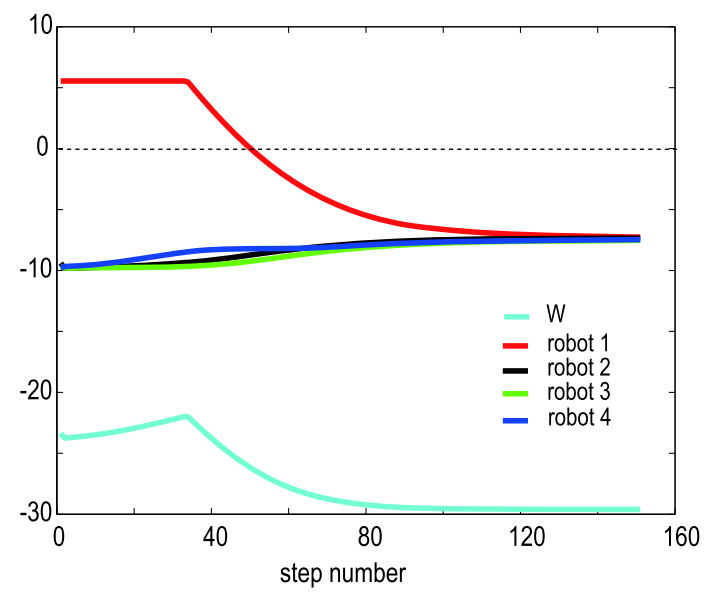

Fig. 11 Total and local objective function

initially placed at the right and top region of the ellipsoidal object. The circles in the figure indicate the final positions of the robots. It can be seen that the robots surround the object and achieve the force-closure condition. The total objective function $W_{G}$ and the local objective functions $f_{i}, i=1, \ldots, 4$ are shown in Fig. 11. It can be seen that $W_{G}$ decreases and all become negative after 50 time steps. This indicates that the local force-closure condition for each robot defined by (4.1) was achieved. It can be observed that there are non-smooth changes of $f_{i}$ in Fig. 11 around the 35th step. This was caused by the transition from the openchain form to the circular form.

The simulation results with a hexagonal object and $n=6$ are shown in Figs. 12 and 13. Similarly, the robots are initially placed at the right bottom region on the surface of the object. Judging from the positions of the circles in Fig. 12, it can be observed that a configuration, which does not allow the object to escape in any direction, was achieved after convergence. In Fig. 13, it can be seen that the total objective function is reduced. In this case, many robots are placed close to the edges of the object. Considering the stability of the grasp, the concentration of robots at particular edges 


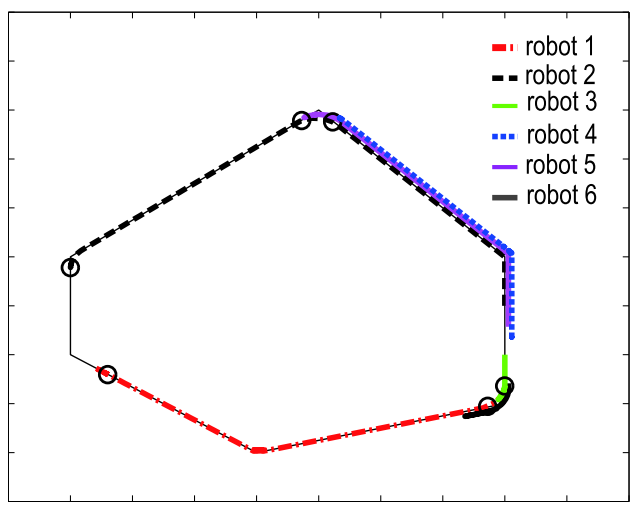

Fig. 12 Trajectories of robots with a hexagonal object

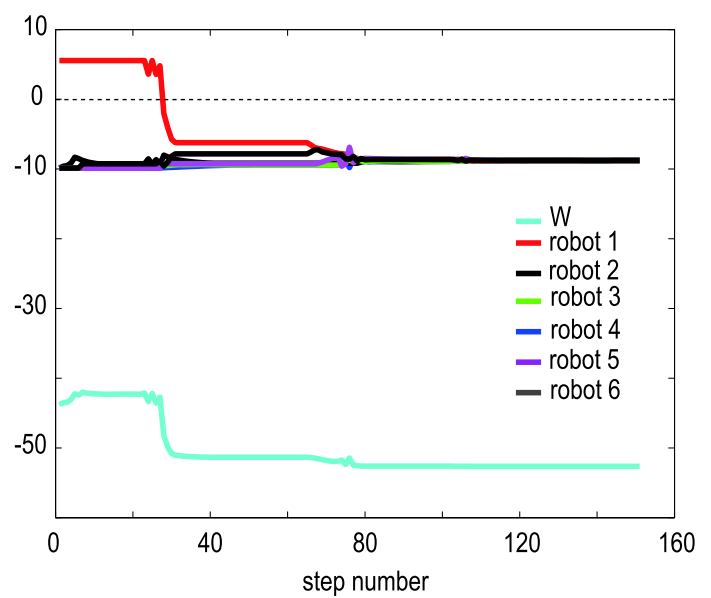

Fig. 13 Total and local objective functions

is obviously undesirable. In our framework, however, only translational motion is taken into account. That is, the configuration obtained by the proposed control is stable in the sense that translational motions of the object can be effectively restrained, but is not sufficiently robust to rotational motions. More extensive definitions of the evaluation function may improve this process.

To verify the convergence property, we simulated the grasping task with different initial conditions. The initial positions of the robots were decided randomly on the surface of ellipsoidal and hexagonal objects. The number of steps required for convergence was evaluated. The condition for convergence is that average motion $\frac{1}{n} \sum_{i=1}^{n} \dot{\boldsymbol{x}}_{i}$ is less than a threshold value $\varepsilon=0.0001$. Table 1 shows the average and the standard deviation over 20 trials for each object. The number of robots is 6 for both objects. In all trials, the robots finally converged to a configuration with the same function value of $W$. Although the steps required for convergence were different according to the initial configurations, the goal of the grasping task was achieved in all cases.

The proposed control scheme has an advantage over an autonomous decentralized system. That is, it is adaptable to
Table 1 Number of steps required for convergence

\begin{tabular}{llc}
\hline Object type & Ave. \# of steps & std. dev. \\
\hline Ellipsoidal object & 306.15 & 116.33 \\
Hexagonal object & 326.65 & 191.39 \\
\hline
\end{tabular}

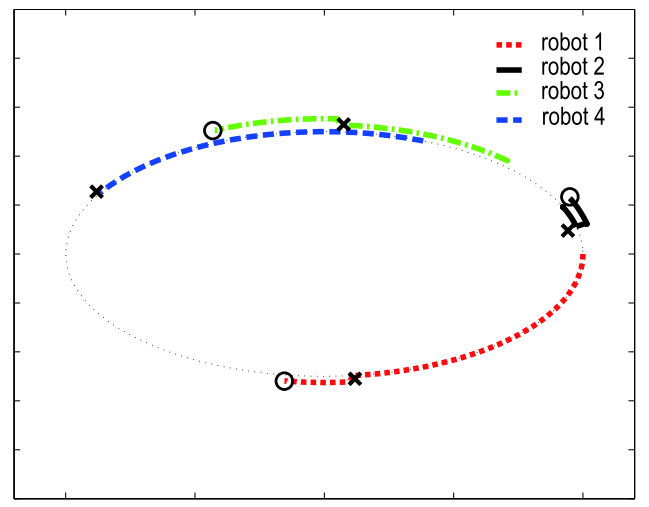

Fig. 14 Trajectories of robots with robot removal

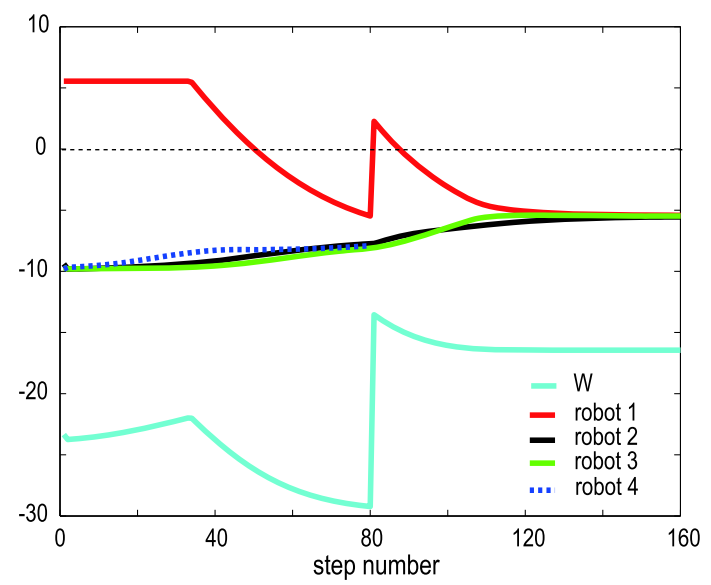

Fig. 15 Total and local objective functions

a change in the number of elements in the system. The next simulation was conducted to verify this adaptability to system size changes. Figures 14 and 15 show the results with four robots, but one robot was removed at the 80th step. The crosses in Fig. 14 indicate the positions of the robots when one robot was removed. Each robot modifies its configuration adaptively to the change in the total number of robots.

Figures 16 and 17 show the results with five robots, where an additional robot was added close to robot 5 at the 80th step. The crosses for robot 2 and robot 4 were omitted because their movements after the addition of the robot were small. It can be observed that the configuration of the robots was modified as the robots around the newly added robot spread out, which is the opposite behavior of the previous simulation where a robot was removed. 


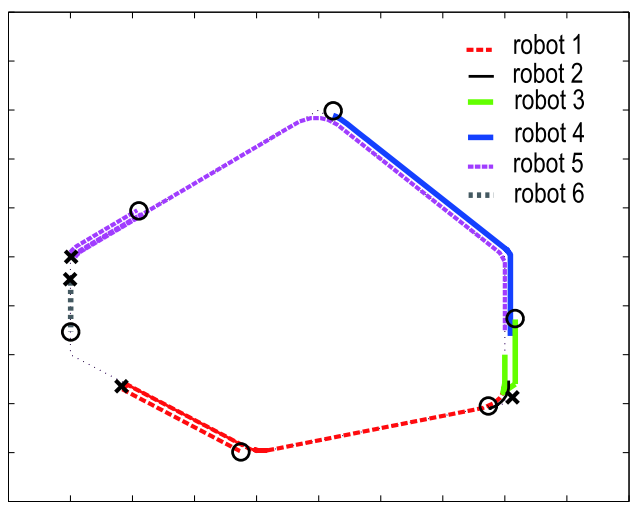

Fig. 16 Trajectories of robots with a robot addition

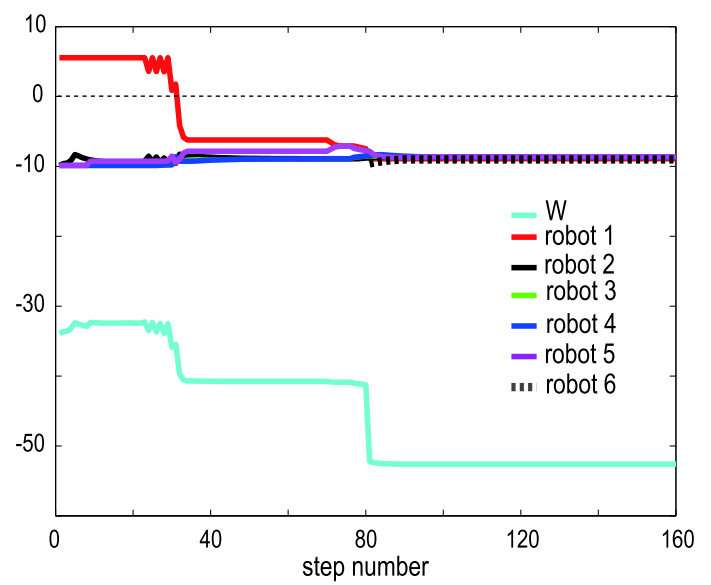

Fig. 17 Total and local objective functions

\section{Experiment}

This section describes an implementation of enclosure behavior through an experiment using multiple decentralized mobile robots. Figure 18 shows the mobile robot of RoboE by Robos corp. The robot has an omni-directional color CCD camera $(120 \times 120$ pixels $)$ mounted at the top. Image information is processed by a CPU (SH-4) and the result is transferred to the other CPU (SH-2) that controls the wheels. The position of the robot is controlled by two parallel wheels.

The object is equipped with green LEDs and each robot is equipped with orange LEDs at the bottom (close to the ground). The robot can identify the object by the color but cannot identify each robot, that is, it can only know that there is another robot. Using color information, green pixels and orange pixels are clustered. The omni-directional visual field is divided by a line that connects the origin of the image and the center of the green cluster. In each region, the center of the orange cluster is measured and is used to determine the position of a neighboring robot.

Owing to the non-holonomic constraint, it is not possible to process arbitrary robot movements. Instead, a point that
Fig. 18 Mobile robot Robo-E
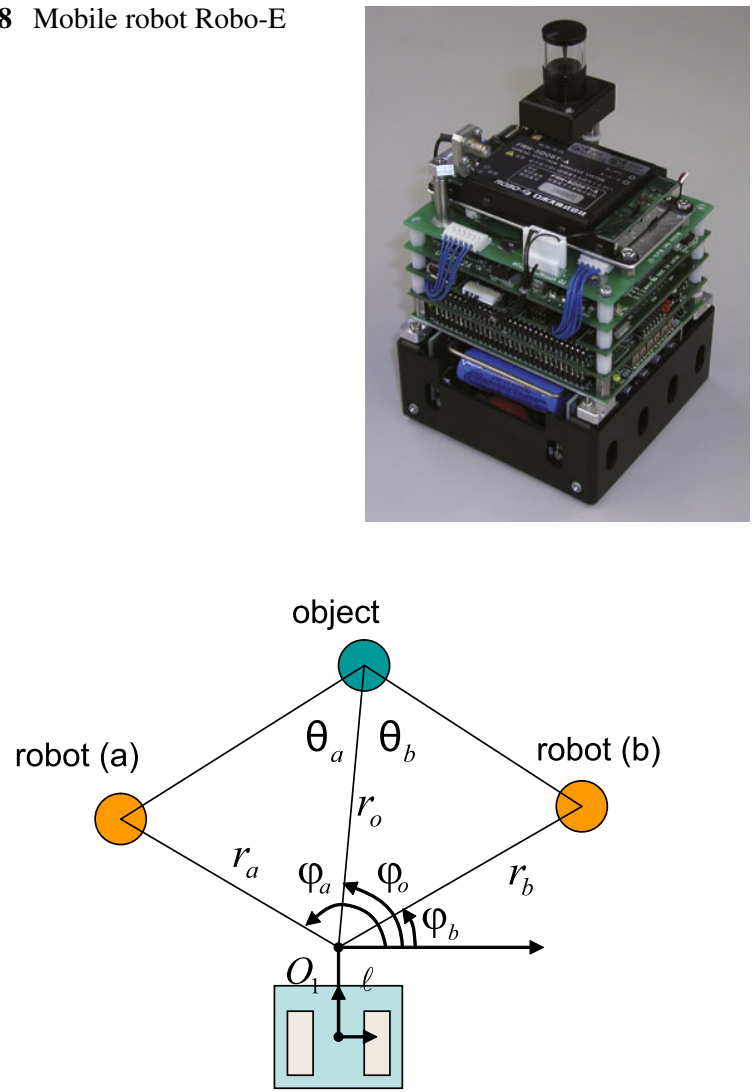

Fig. 19 Local coordinates of robot $i$

has displacement of $\ell$ from the origin of the robot is controlled (e.g., see [26]). Let $O_{1}$ denote the controlled point.

Let $r_{o}, r_{a}$, and $r_{b}$ denote distances from $O_{1}$ to the object, robot on the left side and robot on the right side, respectively. $\psi_{o}, \psi_{a}$, and $\psi_{b}$ denote the angles of the object, the robot on the left side, and the robot on the right side, respectively. $\theta_{a}$ indicated in Fig. 19 can be calculated as

$\theta_{a}=\arctan \left(\frac{r_{a} \sin \left(\psi_{a}-\psi_{o}\right)}{r_{o}-r_{a} \cos \left(\psi_{a}-\psi_{o}\right)}\right)$

using the first law of cosines and the law of sines. Similarly, $\theta_{b}$ can be calculated. $\Delta \theta_{i}$ can be decided by (3.35) as

$\Delta \theta_{i}=-\beta \eta\left(\theta_{i}-\frac{\theta_{i+1}+\theta_{i-1}}{2}\right)=\beta^{\prime}\left(\theta_{a}-\theta_{b}\right)$

$\Delta r_{i}$ can be also decided by (3.34). Displacement in the robot coordinates can be given as

$\Delta x=x_{o}+\left(r_{o}-\Delta r\right) \cos \left(\psi_{o}+\pi+\Delta \theta_{i}\right)$,

$\Delta y=y_{o}+\left(r_{o}-\Delta r\right) \sin \left(\psi_{o}+\pi+\Delta \theta_{i}\right)$,

where $\left(x_{o}, y_{o}\right)$ denotes the position of the object in the robot coordinates. The rotational speed of two wheels $\omega_{1}, \omega_{2}$ that 
realizes the displacement $(\Delta x, \Delta y)$ can be determined as

$$
\left[\begin{array}{c}
\omega_{1} \\
\omega_{2}
\end{array}\right]=\frac{2}{R_{w}}\left[\begin{array}{cc}
-\ell / R_{S} & \ell / R_{S} \\
1 & 1
\end{array}\right]^{-1}\left[\begin{array}{l}
\Delta x \\
\Delta y
\end{array}\right],
$$

where $R_{w}$ and $R_{s}$ denote the radius of the wheel and the distance from the wheel to the midpoint of the shaft, respectively. This rotational speed is given to the robot as a control command.

Figure 20 shows the trajectories of four robots. In this experiment, the object was fixed. The robots at both ends of the open chain recognize that they are at the ends because a neighboring robot can be detected only on one side of the divided regions in the omni-directional images. The robots at the ends moved to spread the formation based on (3.19) and (3.20). It was also possible to demonstrate enclosing behavior using three robots. The robot system is completely decentralized and thus it can adaptively change behavior according to the number of robots used.

\section{Conclusion}

In this paper, we have presented a design for the capturing behavior of an object with decentralized control. The local control policy can be similarly expressed both in the enclosing and grasping tasks, although the coordinate systems used are different. A decentralized form of force-closure conditions were derived for designing the objective function for the grasping task. One of the advantages of the proposed decentralized control is that each robot can judge whether the force-closure condition is achieved by using locally observed information. The proposed local control policies worked effectively in simulations in both tasks. The enclosing behavior was implemented using multiple mobile robots with local observation via omni-directional CCD cameras.

We assumed that there is no obstacle in the environment. When we apply the proposed framework to an environment with obstacles, techniques of detecting moving obstacles
Fig. 20 Trajectories of four robots
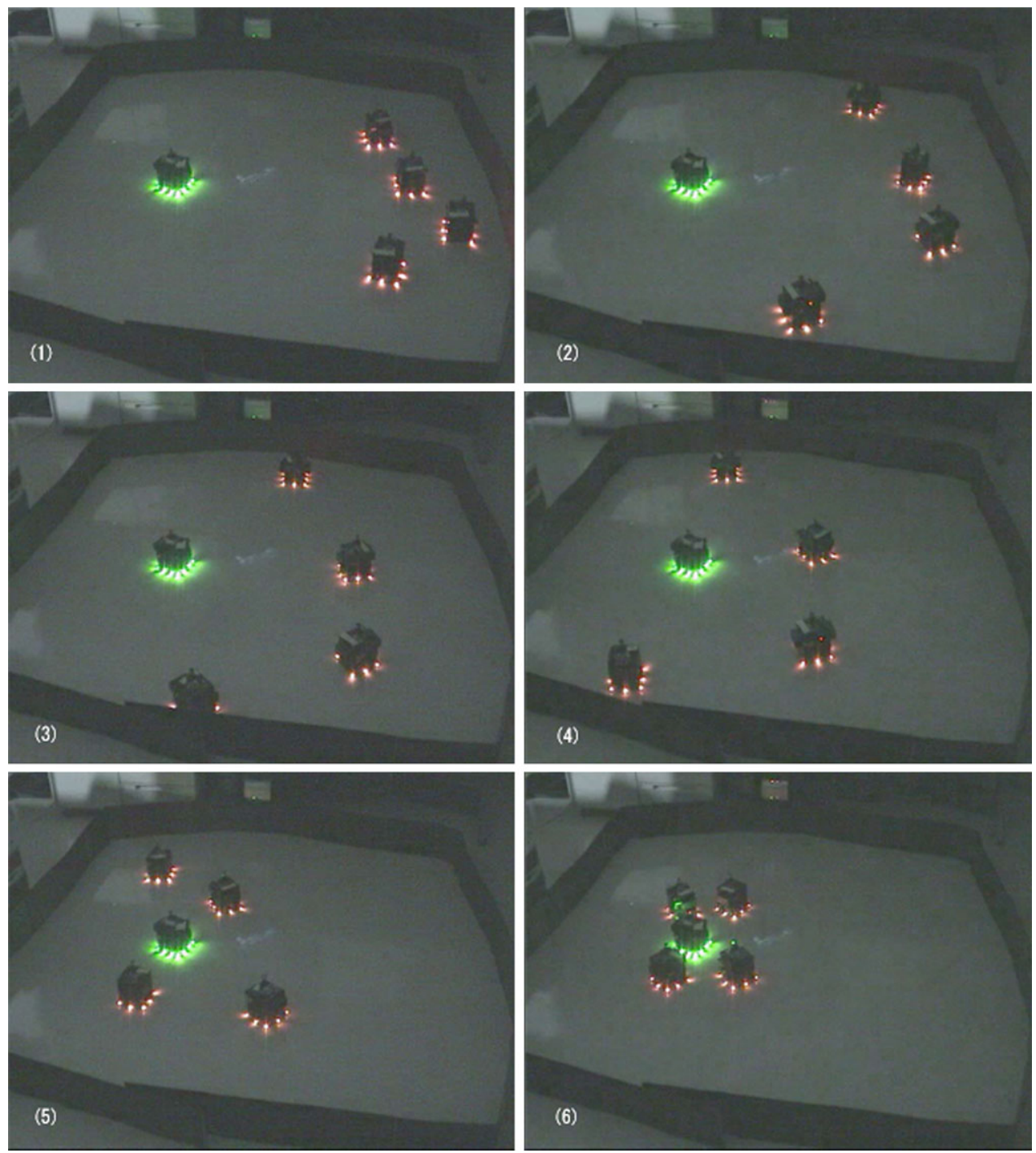
$[13,23]$ will be required to be implemented. Local coordination of behaviors between enclosing and collision avoidance will be also an important issue. To demonstrate the grasping behavior of real robots, it will be necessary to develop methods for measuring local object shape and for constructing control strategies for maneuvering around the surface of objects. Switching or smooth connection of enclosure and grasping are also topics for future study.

Our ideas for building a control policy were (1) to select a coordinate system suitable for accomplishing the task, (2) to derive a local condition that is related to the total objective and to construct an evaluation function, and (3) to approximate unknown information about the environment and the object using locally observed information. We expect that these ideas may also be important to other decentralized control applications in different areas.

Acknowledgements We thank Mr. Kyouji Otsubo and Mr. Yukio Noda for their unstinting assistances in robot experiments and simulations.

Open Access This article is distributed under the terms of the Creative Commons Attribution Noncommercial License which permits any noncommercial use, distribution, and reproduction in any medium, provided the original author(s) and source are credited.

\section{Appendix: Stability of Matrix $-B$ of Difference System}

Here the stability of matrix $-B$ defined in (3.29) is considered. To show the stability, the positive definiteness of $B$ will be checked. The quadratic form $\boldsymbol{x}^{T} B \boldsymbol{x}$ can be calculated as

$$
\begin{aligned}
\boldsymbol{x}^{T} B \boldsymbol{x}= & 2\left\{x_{1}^{2}+x_{2}^{2}+\cdots+x_{n}^{2}-x_{1} x_{2}-x_{2} x_{3}\right. \\
& \left.-\cdots-x_{n-1} x_{n}\right\} \\
= & \left\{a_{1}\left(x_{1}-\frac{x_{2}}{2}\right)^{2}+a_{2}\left(x_{2}-\frac{2}{3} x_{3}\right)^{2}\right. \\
& +a_{3}\left(x_{4}-\frac{4}{5} x_{5}\right)^{2}+a_{4}\left(x_{5}-\frac{5}{6} x_{6}\right)^{2} \\
& \left.+\cdots+a_{n-2}\left(x_{n-1}-\frac{x_{n}}{2 a_{n-2}}\right)\right\},
\end{aligned}
$$

where

$a_{1}=1, \quad a_{2}=\frac{3}{4}, \quad a_{3}=\frac{5}{8}, \quad a_{4}=\frac{3}{5}, \ldots$

and the coefficient series $\left\{a_{k}\right\}$ satisfies the following relation;

$a_{k+1}=1-\frac{1}{4 a_{k}}, \quad k=1, \ldots, n-3$.
It can be easily seen that

$a_{k}>\frac{1}{2} \Rightarrow a_{k+1}=1-\frac{1}{4 a_{k}}>\frac{1}{2}, \quad k=1, \ldots, n-3$,

which means that all of the coefficients are positive. Thus, matrix $-B$ is stable because $B$ is positive definite.

\section{References}

1. Ayanian N, Kumar V (2010) Decentralized feedback controllers for multiagent teams in environments with obstacles. IEEE Trans Robot 26(5):878-887

2. Balch T, Arkin RC (1998) Behavior-based formation control for multiagent robot teams. IEEE Trans Robot Autom 14(6):926-939

3. Chung TH, Burdick JW, Murray RM (2006) A decentralized motion coordination strategy for dynamic target tracking. In: Proc of the 2006 IEEE int conf on robotics and automation, pp 2416-2422

4. Cortes J, Martinez S, Karatas T, Bullo F (2004) Coverage control for mobile sensing networks. IEEE Trans Robot Autom 20(2):243-255

5. Desai JP, Ostrowski JP, Kumar V (2001) Modelling and control of formation of nonholonomic mobile robots. IEEE Trans Robot Autom 17(6):905-908

6. Elara MR, Wijesoma WS, Calderon CAA, Zhou C (2009) Experimenting false alarm demand for human robot interactions in humanoid soccer robots. Int J Soc Robot 1(2):171-180

7. Hsieh M-yA, Kumar V (2006) Pattern generation with multiple robots. In: Proc of the 2006 IEEE int conf on robotics and automation, pp 2442-2447

8. Inagaki S, Yuasa H, Arai T (2003) CPG model for autonomous decentralized multi-legged robot system-generation and transition of oscillation patterns and dynamics of oscillators. Robot Auton Syst 44:171-179

9. Lin Z, Broucke M, Francis B (2004) Local control strategies for groups of mobile autonomous agents. IEEE Trans Autom Control 49(4):622-629

10. Liu G, Xu J, Li Z (2004) On quality functions for grasp synthesis fixture planning and coordinated manipulation. IEEE Trans Autom Sci Eng 1(2):146-162

11. Mataric MJ, Sukhatme GS, Ostergaard EH (2003) Multi-robot task allocation in uncertain environments. Auton Robots 14(23):255-263

12. Murray R, Li Z, Sastry S (1994) A mathematical introduction to robotic manipulation. CRC Press, Boca Raton

13. Nair D, Aggarwal JK (1998) Moving obstacle detection from a navigating robot. IEEE Trans Robot Autom 14(3):404-416

14. Pereira GAS, Kumar V, Campos MFM (2004) Decentralized algorithms for multi-robot manipulation via caging. Int J Robot Res 23(7):783-795

15. Reynolds CW (1987) Flocks, herds, and schoolds: a distributed behavioral model. Comput Graph 21(4):25-34

16. Roumeliotis SI, Bekey GA (2002) Distributed multirobot localization. IEEE Trans Robot Autom 18(5):781-795

17. Song P, Kumar V (2002) A potential field approach to multi-robot manipulation. In: Proc of the IEEE int conf on robotics and automation, pp 1217-1222

18. Stirling WC, Nokleby MS (2009) Satisficing coordination and social welfare for robotic societies. Int J Soc Robot 1(1):53-69

19. Tsujita K, Tsuchiya K, Onat A (2003) Decentralized autonomous control of a quadruped locomotion robot. Artif Life Robot 5:152158 
20. Ulam P, Balch T (2004) Using optimal foraging models to evaluate learned robotic foraging behavior. Adapt Behav 12(4):213-222

21. Wagner I, Bruckstein AM (1997) Rowstraightening by local interactions. Circuits Syst Signal Process 16(3):287-305

22. Wang Z, Takano Y, Hirata Y, Kosuge K (2004) A pushing leader based decentralized control method for cooperative object transportation. In: Proc of IEEE/RSJ international conference on intelligent robots and systems, vol 1, pp 1035-1040

23. Wu M, Sun JY (2010) Moving object detecting and tracking with mobile robot based on extended Kalman filter in unknown environment. In: Proc of 2010 international conference on machine vision and human-machine interface, pp 64-67

24. Yamaguchi H (2003) A distributed motion coordination strategy for multiple nonholonomic mobile robots in cooperative hunting operations. Robot Auton Syst 43:257-282

25. Yuasa H, Ito M (1999) Self-organizing system theory by use of reaction-diffusion equation on a graph. In: Proc of IEEE int conf on systems, man and cybernetics, vol 1, pp 211-216

26. Yun X, Yamamoto Y (1993) Internal dynamics of a wheeled mobile robot. In: Proc of the IEEE/RSJ international conf on intelligent robots and systems, pp 1288-1294

27. Zhu X, Wang J, Hirzinger G (2003) Synthesis of force-closure grasps on 3-d objects based on q distance. IEEE Trans Robot Autom 19(4):669-678
Yuichi Kobayashi received the B.E., M.E. and Ph.D. degrees from The University of Tokyo, Japan in 1997, 1999 and 2002, respectively. Since 2002 he has been a research scientist at Bio-mimetic Control Research Center in RIKEN, Motor Systems Control Theory Laboratory. He is currently working as an Associate Professor at Department Electrical and Electronic Engineering, Faculty of Engineering, Tokyo University of Agriculture and Technology from 2007. His research interests include adaptation, recognition and motion learning of robots.

Shigeyuki Hosoe received the M.S. degree in Metallurgical Engineering, in 1967, and the D. of Eng. degree in Applied Physics, in 1973, from Nagoya University, Nagoya Japan. He held positions at the Faculty of Engineering, Nagoya University, from 1976 to 2006, where he is now a Professor Emeritus. He was a Head of the Motor Systems Control Theory Laboratory, Bio-Mimetic Control Research Center, RIKEN, Japan from 1999 to 2008, and was a Director of the Center from 2001 to 2008 . He is currently a Director of RIKEN-TRI Collaboration Center for Human-Interactive Robot Research. His research interests include the theory for robust control and hybrid control systems, control of bio-mimetic systems, and robotics. 